The Search for Extrasolar Planets

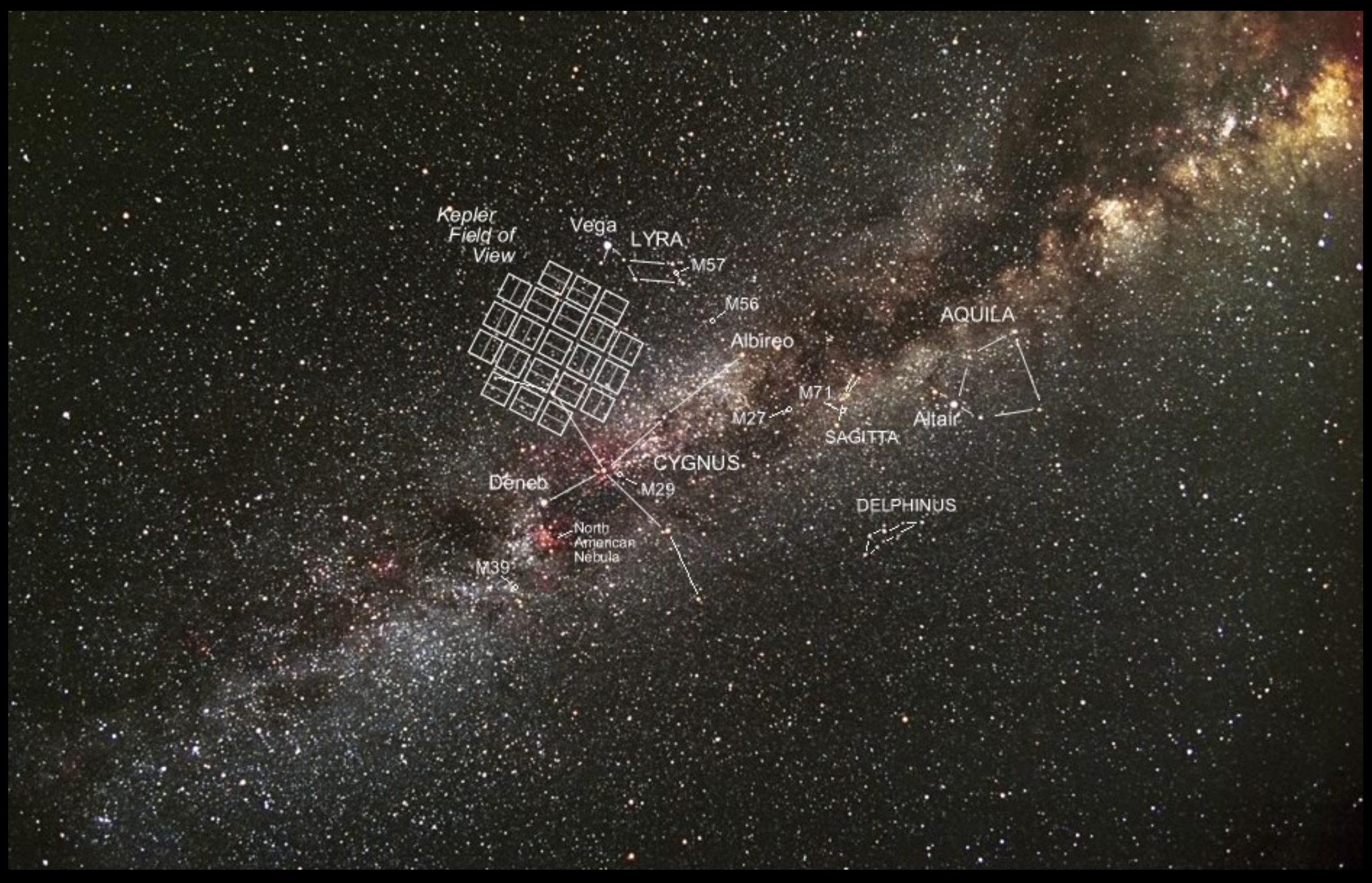

A Presentation and Discussion for the Osher Life-Long-Learning Institute Our Place in the Universe

Mitzi Adams and Dr. Allyn Tennant 


\section{Why Do We Care?}

\section{Frank Drake, 1961}

$N=R^{*} \times f_{p} \times n_{e} \times f_{e} \times f_{i} \times f_{c} \times L$

$N=$ Total number of civilizations in Milky Way

$\mathrm{R}^{*}=$ Rate of Star Formation

$f_{p}=$ fraction of $R$ that have planetary systems

$\mathrm{n}_{\mathrm{e}}=$ number of planets in those systems with habitable zones

$\mathrm{f}_{\mathrm{e}}=$ the fraction of those with life

$f_{i}=$ the fraction of those with intelligent life

$\mathrm{f}_{\mathrm{c}}=$ the fraction of those which create civilizations that advertise their existence

$\mathrm{L}=$ the time those civilizations send signals into space (I Love Lucy)

\section{Sara Seager, 2013 \\ $N=N^{*} \times f_{Q} \times f_{H Z} \times f_{O} \times f_{L} \times f_{S}$}

$\mathrm{N}=$ Number of planets with detectable signs of life

$\mathrm{N}^{*}=$ Number of stars observed

$f_{Q}=$ fraction of those that are quiet

$\mathrm{f}_{\mathrm{HZ}}=$ the fraction of stars with rocky planets in the habitable zone

$f_{O}=$ the fraction of those planets that can be observed

$f_{L}=$ the fraction of those that have life

$f_{s}=$ the fraction of those on which that life produces a detectable signature gas 


\section{History -- Wobbles, Proper Motion}

For stars that are too far away or are too close to each other
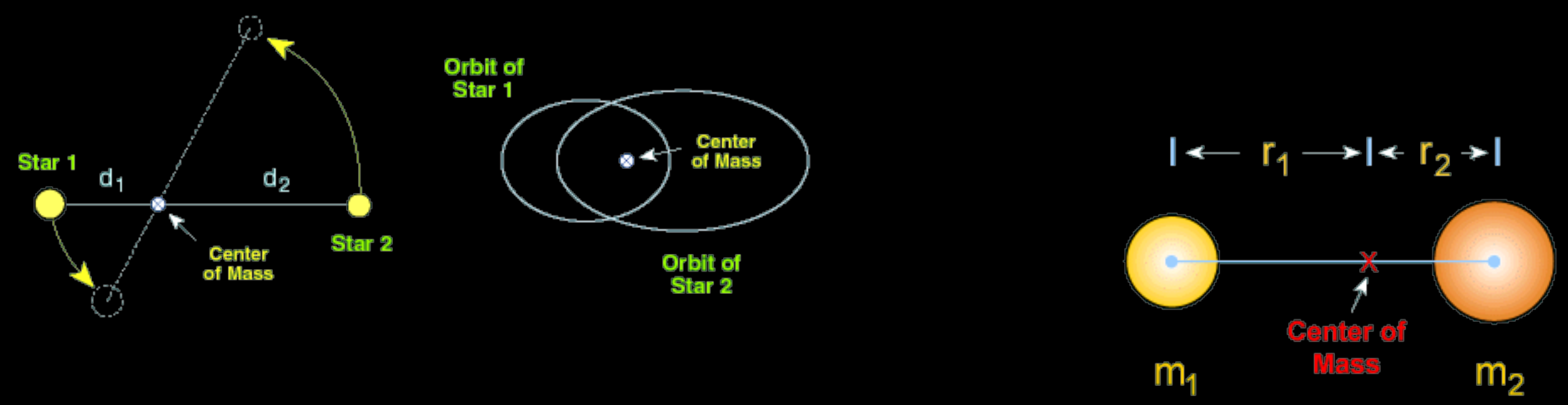

$\left(m_{1}+m_{2}\right) P^{2}=\left(r_{1}+r_{2}\right)^{3}=R^{3}$

$\mathrm{R}=\mathrm{r}_{1}+\mathrm{r}_{2}$

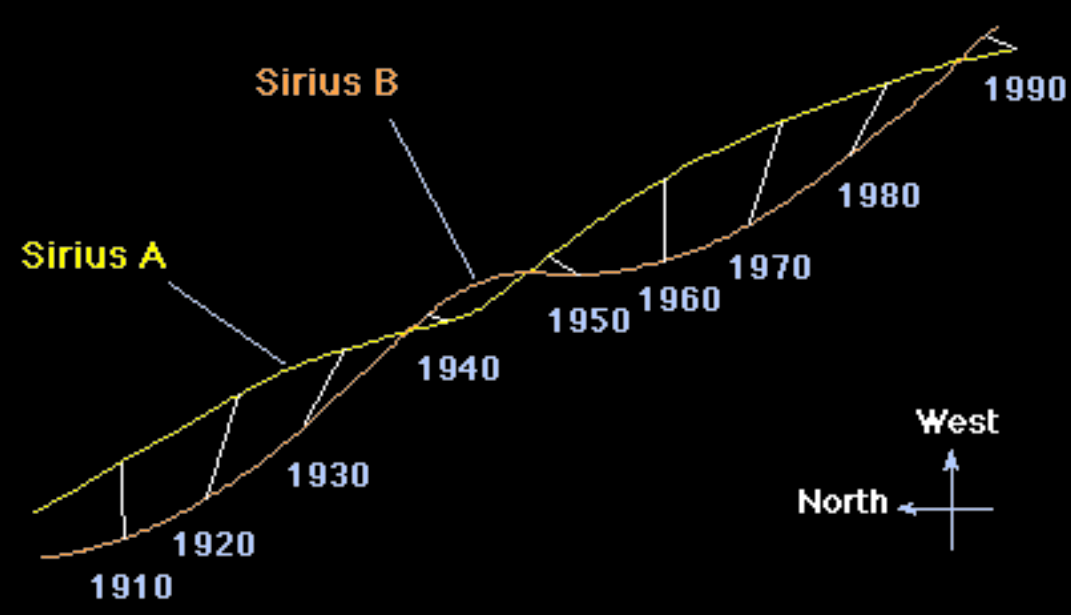

from

Knowing period and average distance, solve for total mass.

http://csep10.phys.utk.edu/astr162/lect/binaries/astrometric.html 


\section{Solar System's Barycenter}

$$
\begin{gathered}
\Phi \sim 1.5 \text { It_sec/ 4_It_year }=1.5 /\left(4^{*} \pi^{*} 1 \mathrm{e}^{7}\right)=10^{-8} \text { radians } \\
\Phi=10^{-8} \text { radians * } 57 \text { deg/radian * } 3600 \text { arcsec } / \text { deg }=2 \mathrm{marcsec}
\end{gathered}
$$

Need dedicated workers for 30 years AND Stable equipment

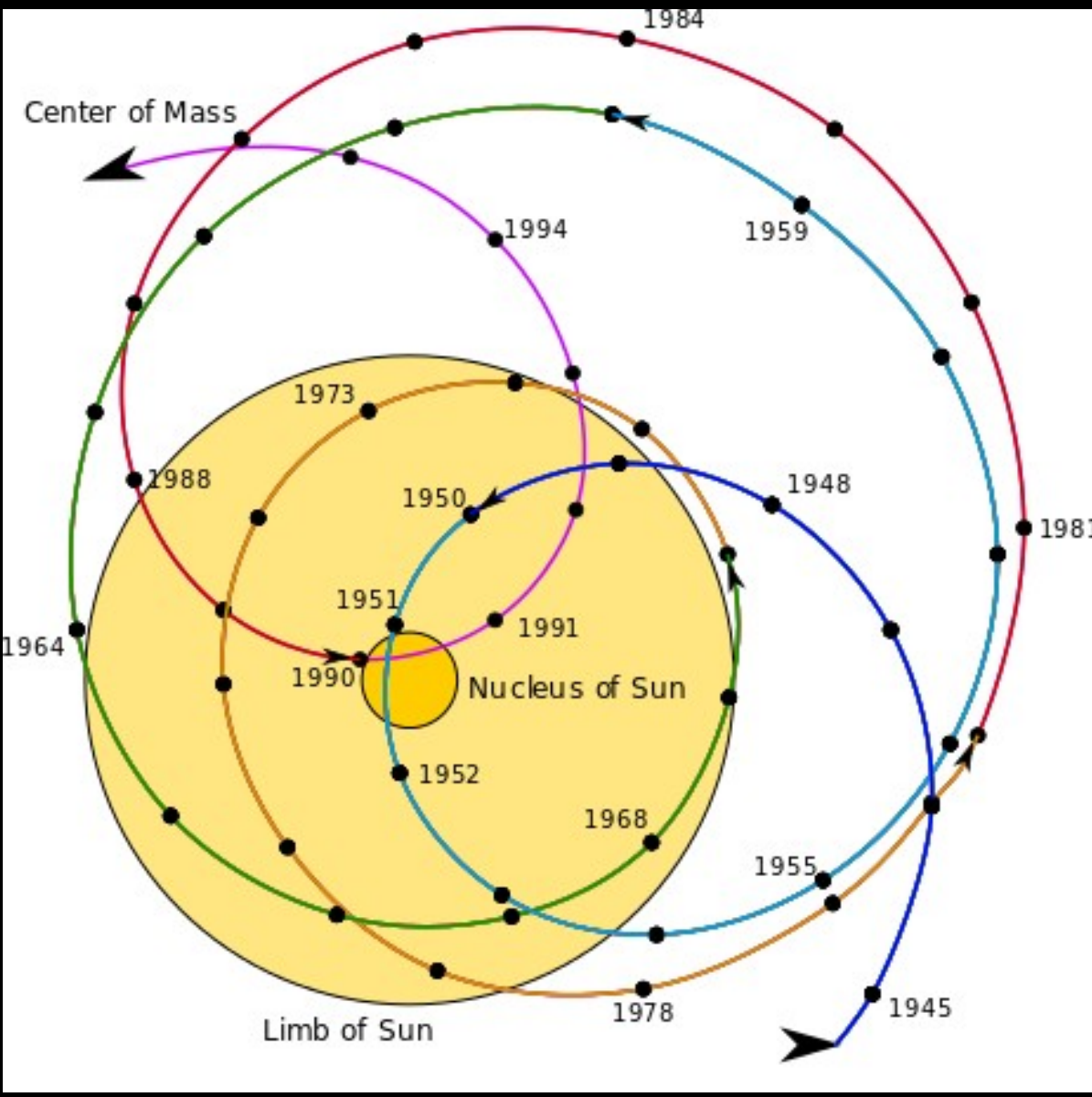

Earth/Moon barycenter is inside Earth, $4671 \mathrm{~km}$ from center

By Solarsystembarycenter.gif: Carl Smithderivative work: Rubik-wuerfel (talk) http://s173.photobucket.com/user/CarlSmith_2007/media/Sun\%20SSB/ssb-orbit-col.gif.html GIF, CC BY-SA 3.0, https://commons.wikimedia.org/w/index.php?curid=9952653 


\section{History -- Wobbles, Doppler Shifts}

Light source moving away from the observer, the observed wavelength will get longer, and hence REDSHIFTED

Light source moving towards the observer, the observed wavelength will get shorter, and hence BLUESHIFTED

$$
\begin{array}{cl}
\lambda_{\mathrm{obs}}-\lambda_{\mathrm{em}}=\mathrm{v} / \mathrm{c} & \lambda_{\mathrm{obs}}=\text { wavelength observed } \\
\lambda_{\mathrm{em}} & \lambda_{\mathrm{em}}=\text { wavelength emitted }
\end{array}
$$
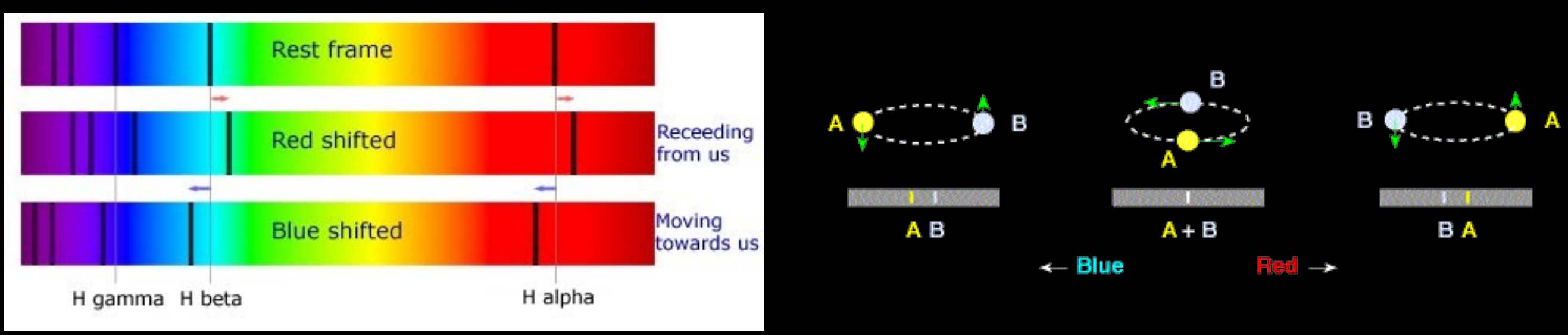


\section{Doppler Spectroscopy}

(continued)

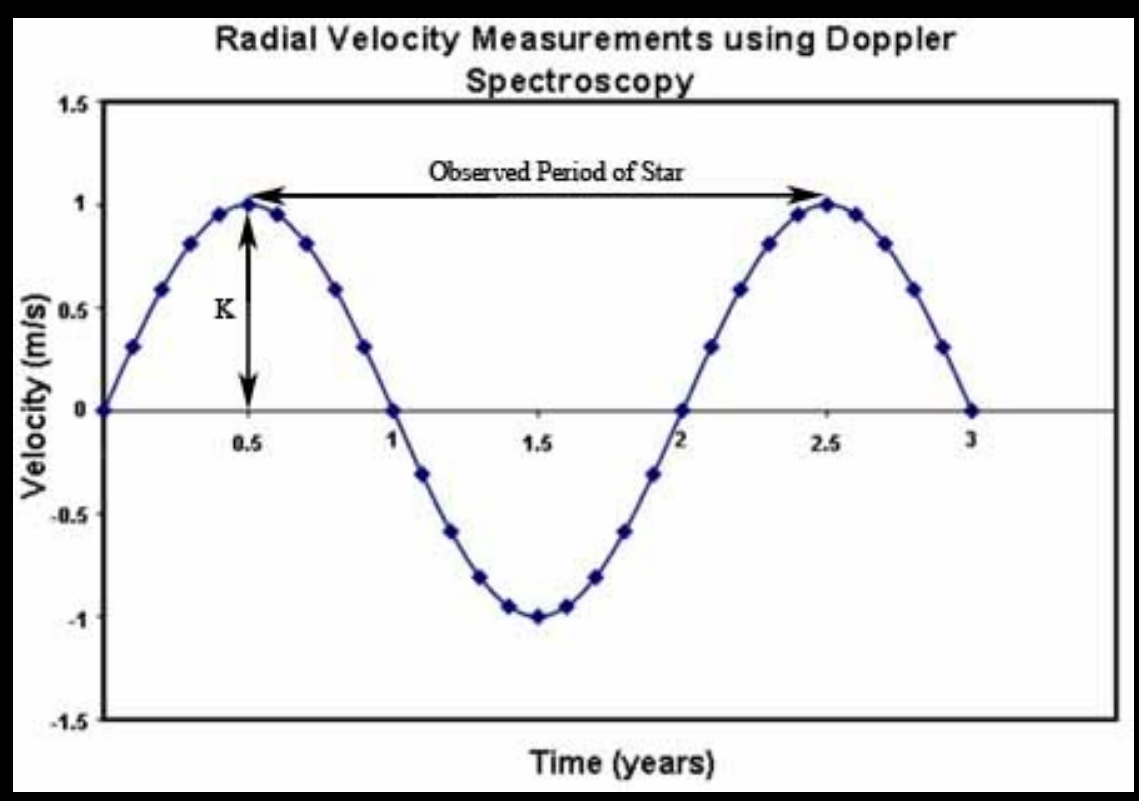




\section{Doppler Spectroscopy}

$$
\mathrm{V} \sim 2^{*} \pi^{*} 1 \mathrm{e}^{6} \mathrm{~km} /\left(10^{*} \pi^{*} 1 \mathrm{e}^{7} \mathrm{sec}\right)=20 \mathrm{~m} / \mathrm{sec}
$$

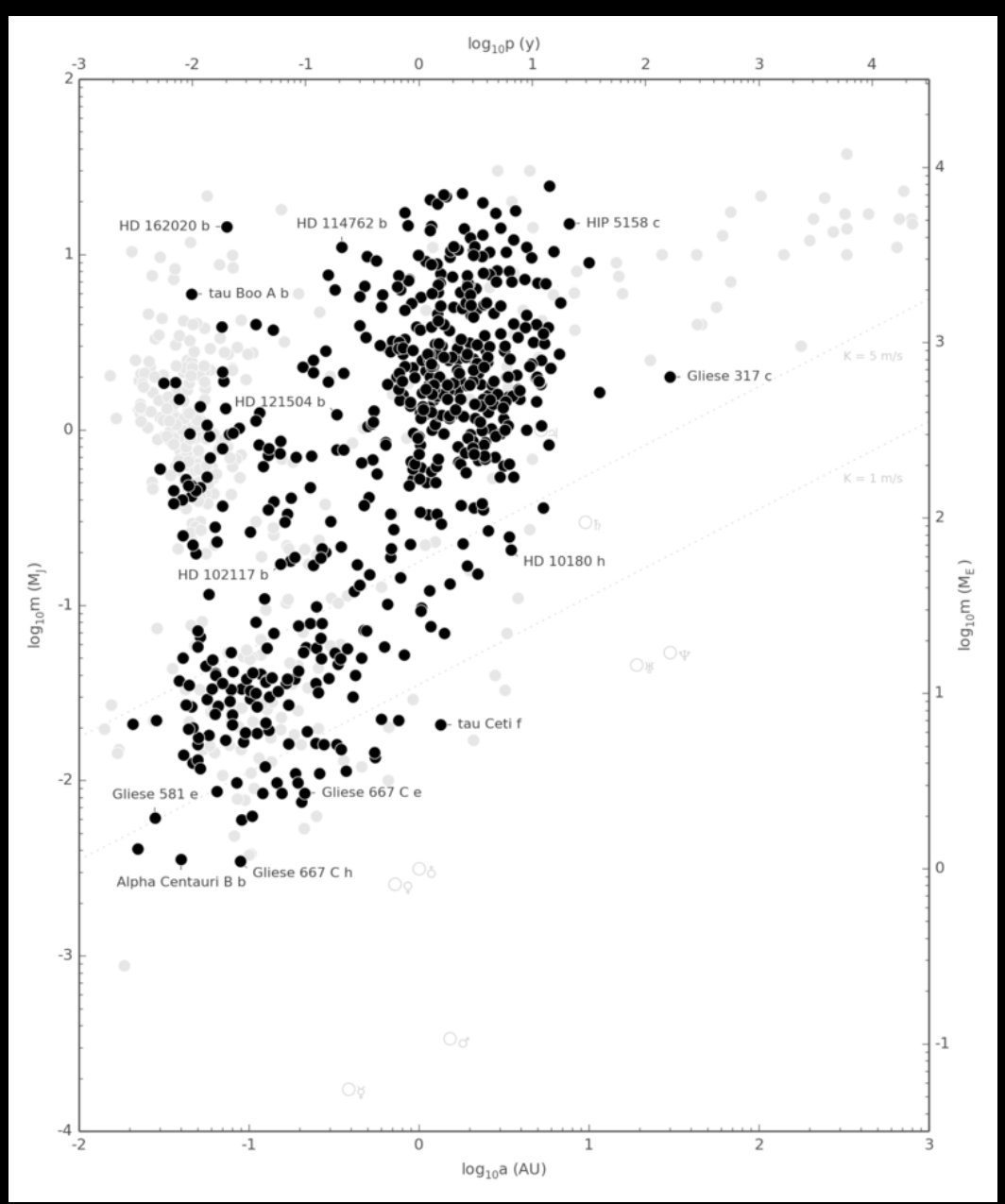

$\begin{array}{lll}\text { Planet Mass } & \begin{array}{c}\text { Distance } \\ \text { AU }\end{array} & \begin{array}{c}\text { Radial velocity } \\ \left(\mathrm{v}_{\text {radial }}\right)\end{array} \\ \text { Jupiter } & 1 & 28.4 \mathrm{~m} / \mathrm{s} \\ \text { Jupiter } & 5 & 12.7 \mathrm{~m} / \mathrm{s} \\ \text { Neptune } & 0.1 & 4.8 \mathrm{~m} / \mathrm{s} \\ \begin{array}{l}\text { Neptune } \\ \text { Super-Earth } \\ (5 \mathrm{M} \oplus)\end{array} & 1 & 1.5 \mathrm{~m} / \mathrm{s} \\ \begin{array}{l}\text { Alpha Centauri Bb } \\ (1.13 \pm 0.09 \mathrm{M} \oplus)\end{array} & 0.1 & 1.4 \mathrm{~m} / \mathrm{s} \\ \begin{array}{l}\text { Super-Earth } \\ (5 \mathrm{M} \oplus)\end{array} & 0.04 & 0.51 \mathrm{~m} / \mathrm{s} \\ \text { Earth } & 1 & 0.45 \mathrm{~m} / \mathrm{s} \\ & 1 & 0.09 \mathrm{~m} / \mathrm{s}\end{array}$

Mass and distance from parent star of planets discovered through 2013 using radial velocity. Light gray dots are planets discovered using other methods.

By Aldaron, a.k.a. Aldaron - Own work, based on a figure by E.A.L. and used with permission, Public Domain, https://commons. wikimedia.org/w/index.php?curid=7410029 
Mercury Transit, May 9, 2016

From http://spaceweathergallery.com, taken by Nunzio Micale in Vieste, Italy. 


\section{Kepler Mission}

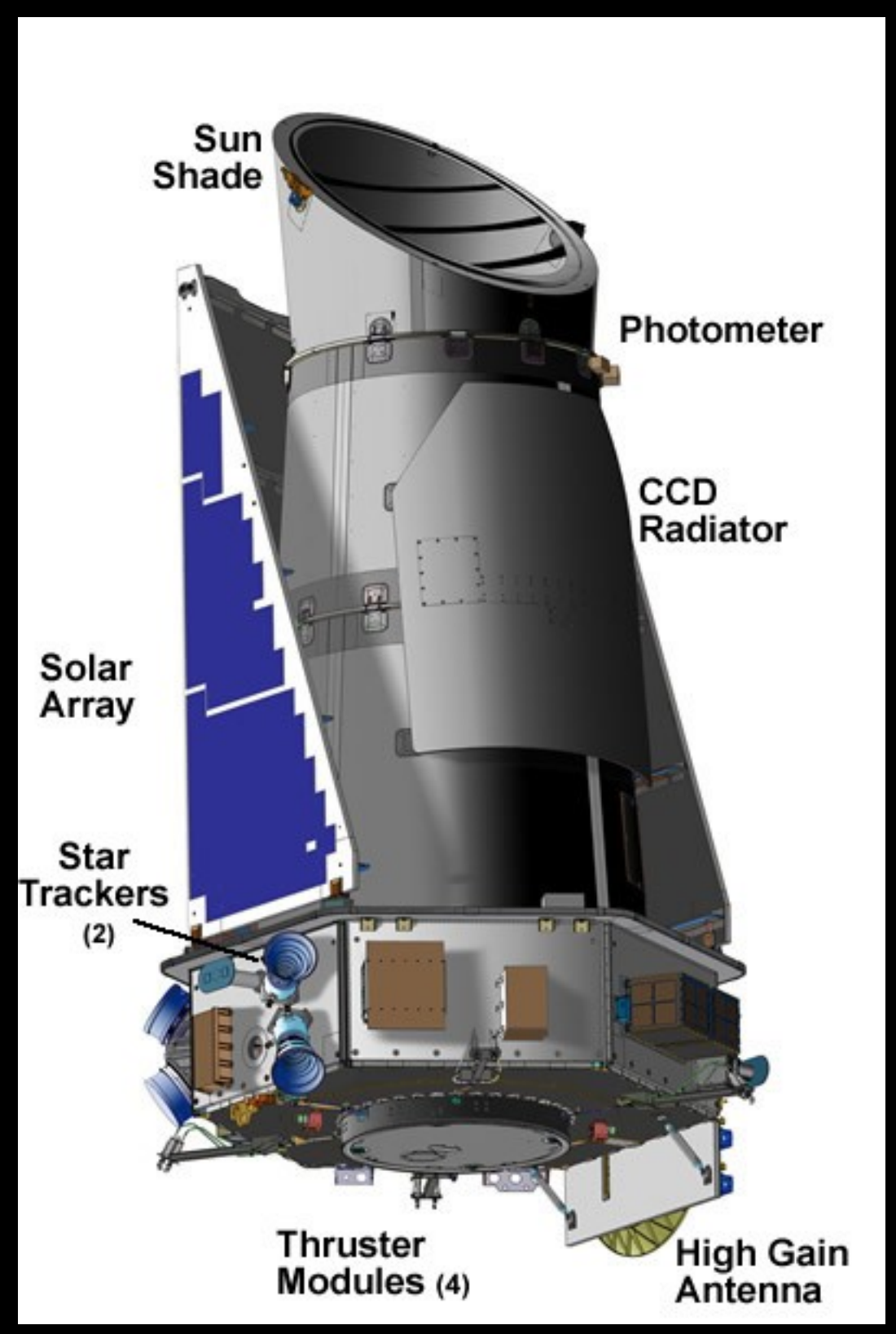

Launched March 6, 2009

- Monitor 100,000 main-sequence stars

- Mission lifetime of 3.5 yrs to 6 yrs

- In Earth-trailing heliocentric orbit

- Stares at same fov over lifetime

- Has a 0.95 m photometer

- Primary mirror: $1.4 \mathrm{~m}$

- Bandpass: 4300-8900 Angstroms

- Extra-solar planet must be edge on to us

- To reliably detect planets in habitable zone, transits are once per year...must have four

- Second reaction wheel failed 2013 May 11, ending the primary mission. 


\section{Kepler Planets Start Out as "Candidates"}

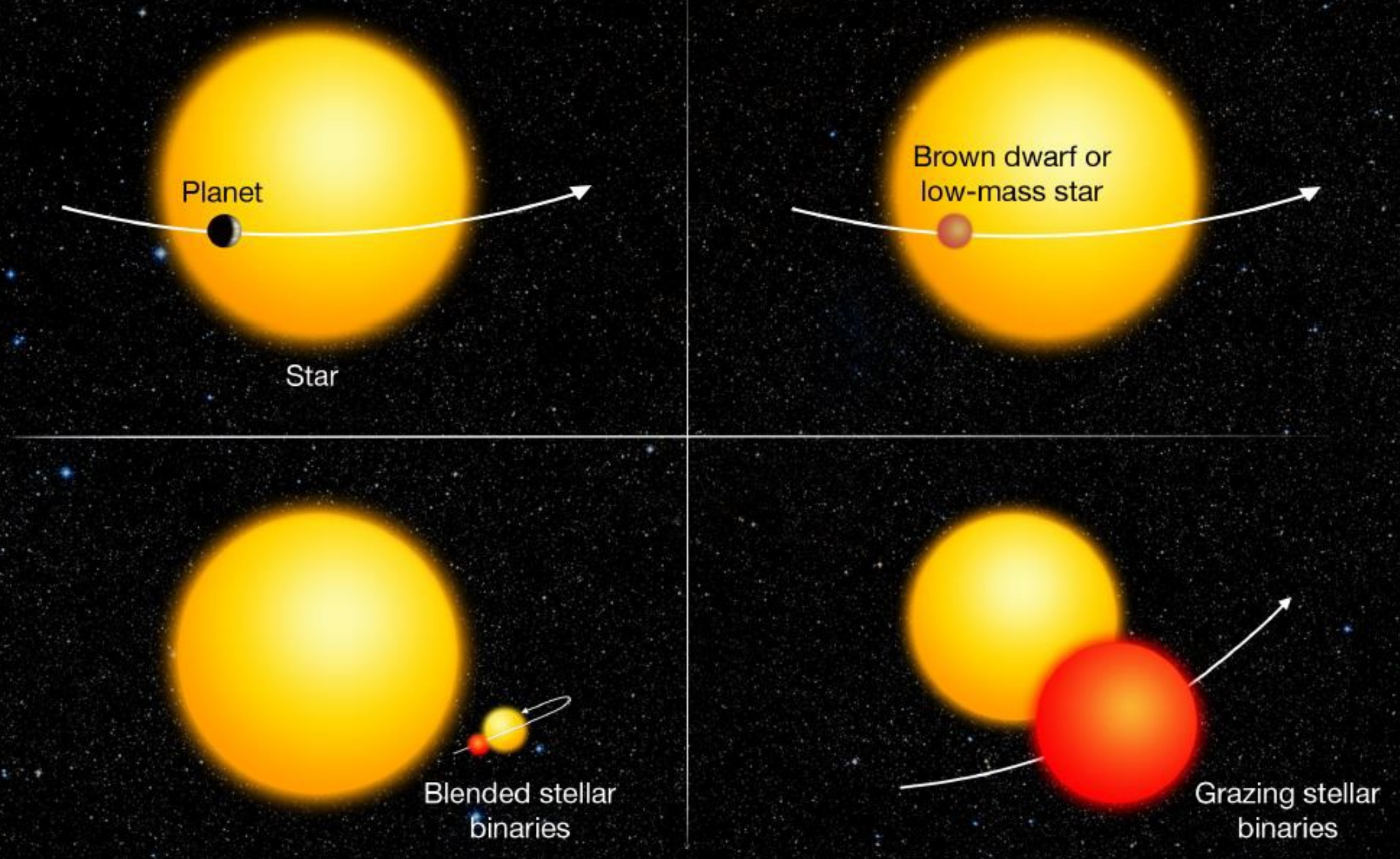




\section{Planet Verification}

Often requires extensive follow-up observations

W. M. KECK OBSERVATORY

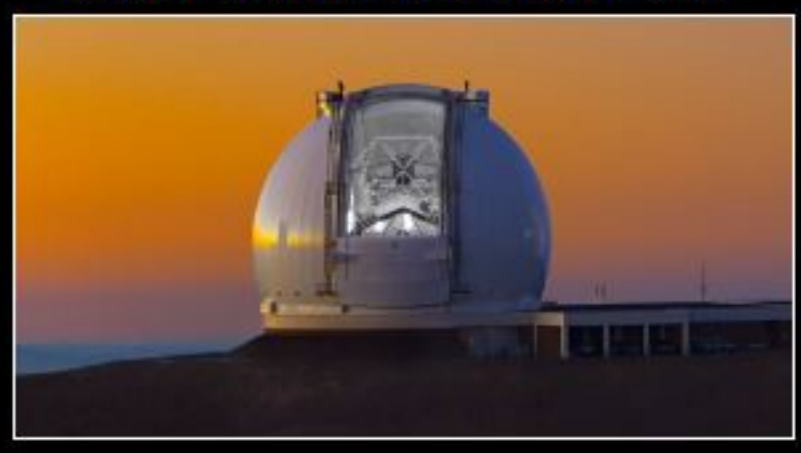

GEMINI NORTH OBSERVATORY

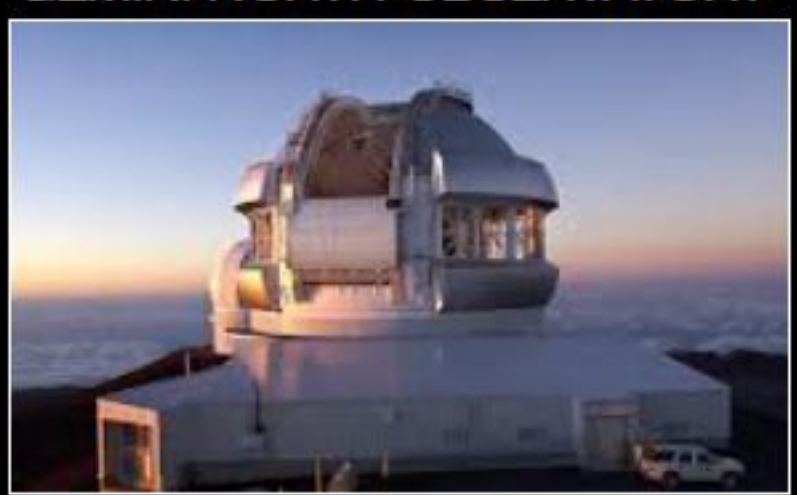

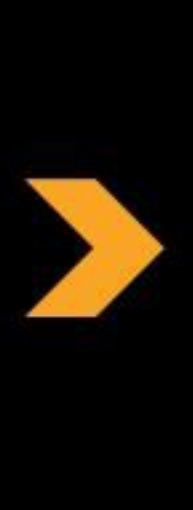

RADIAL VELOCITY

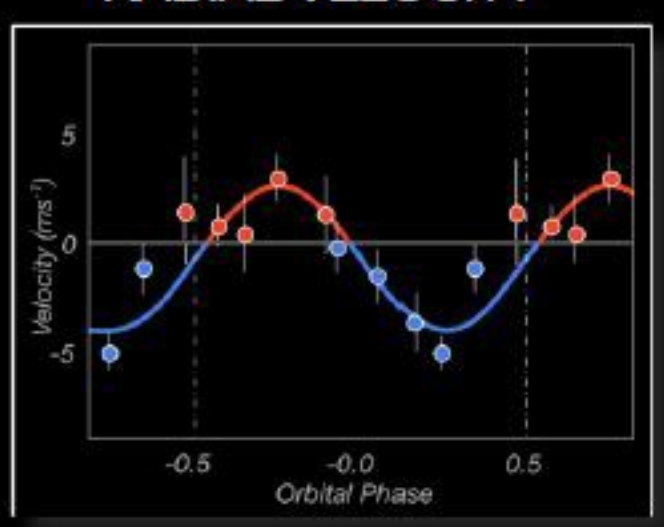

IMAGING

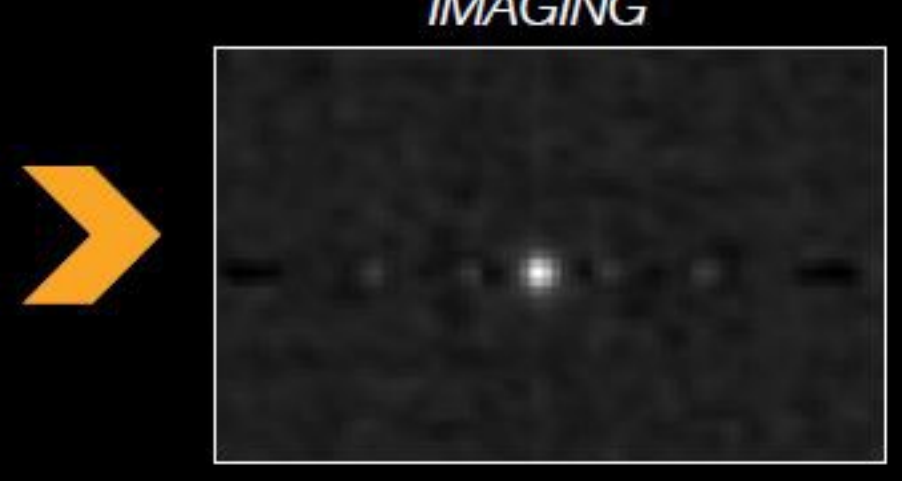

KEPLER-10b
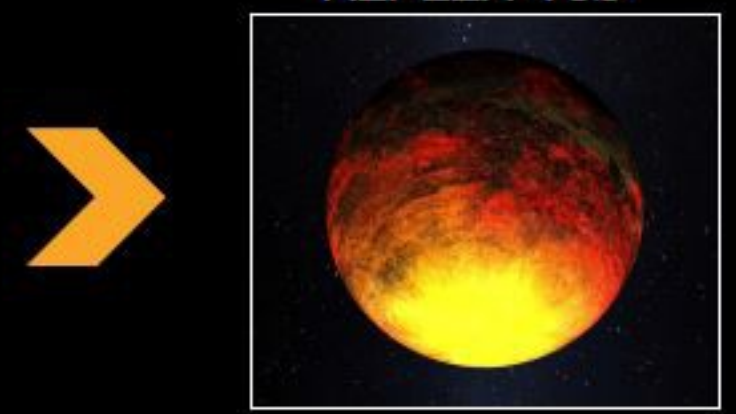

KEPLER-186f
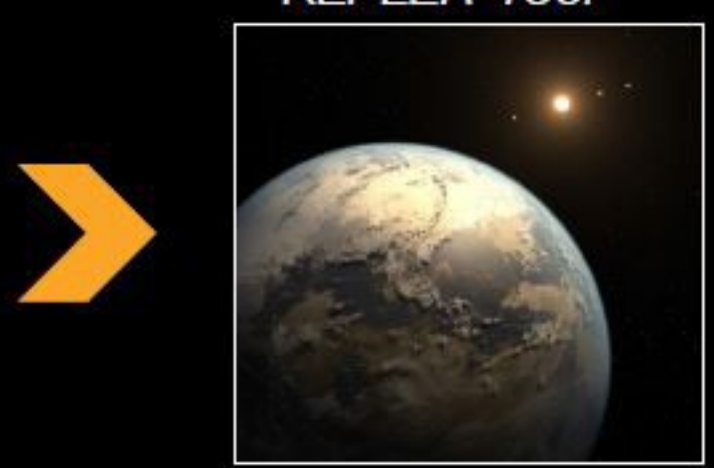


\section{Intriguing Exoplanets}

Kepler-186f, the first rocky planet to be found within the habitable zone, $\sim 500$ ly away.

HD 209458b (Osiris), the first exoplanet to be seen in transit. A hot Jupiter, it is 150 ly away.

Kepler 22b, at 600 ly away, is in the habitable zone of its Sun-like star.

OGLE-2005-BLG-390Lb (Hoth), 5.5 times mass of Earth, 20,000 ly away is NOT in the habitable zone. Hoth's orbit is about three times farther out than Earth.

b

C

HR 8799, at 130 ly away, has four large planets, each 2-3 times the mass of Jupiter.
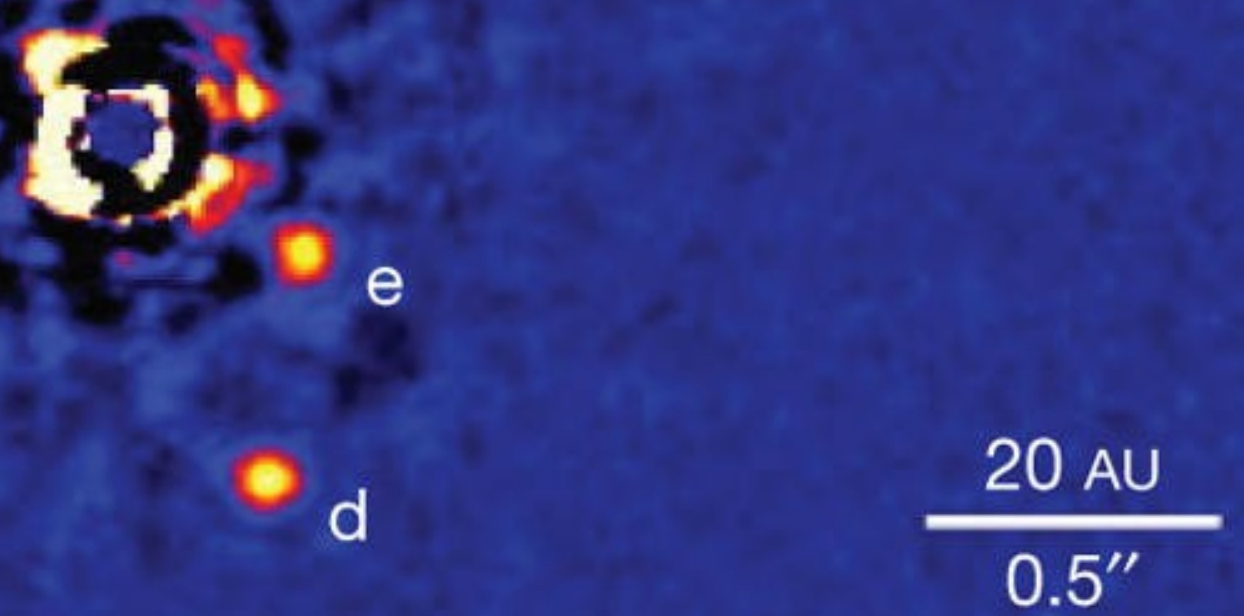


\section{Kepler Mission: Results}

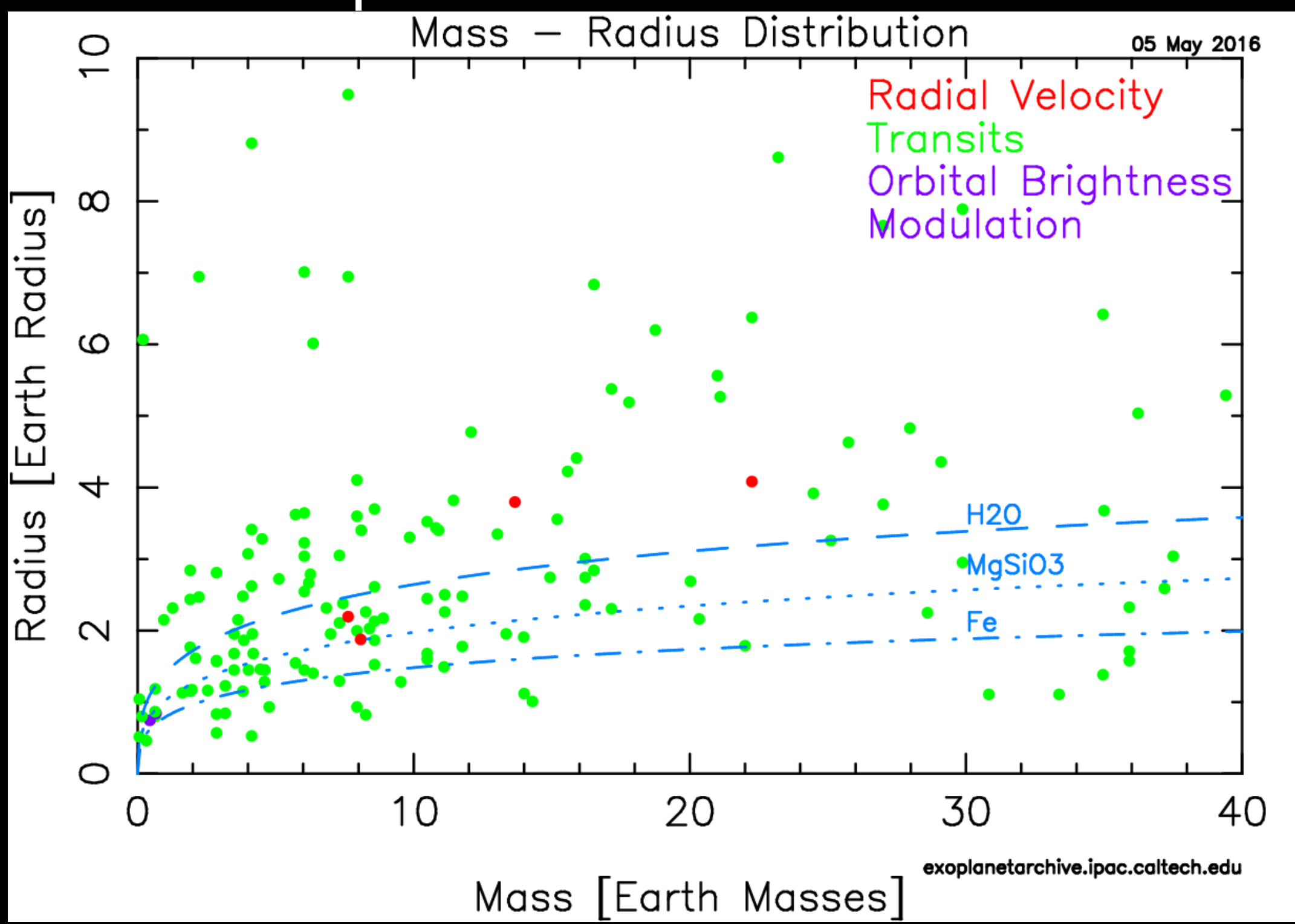

http://kepler.nasa.gov/Mission/discoveries/

1041 Confirmed Planets out of 4706 Candidates

http://exoplanetarchive.ipac.caltech.edu/videos/koi-radiusvperiod-nexsci.mp4 http://exoplanetarchive.ipac.caltech.edu/videos/mass_period_movie_nexsci.mp4 http://exoplanetarchive.ipac.caltech.edu/videos/exo_discovery_histogram.mp4 
Kepler-452 System

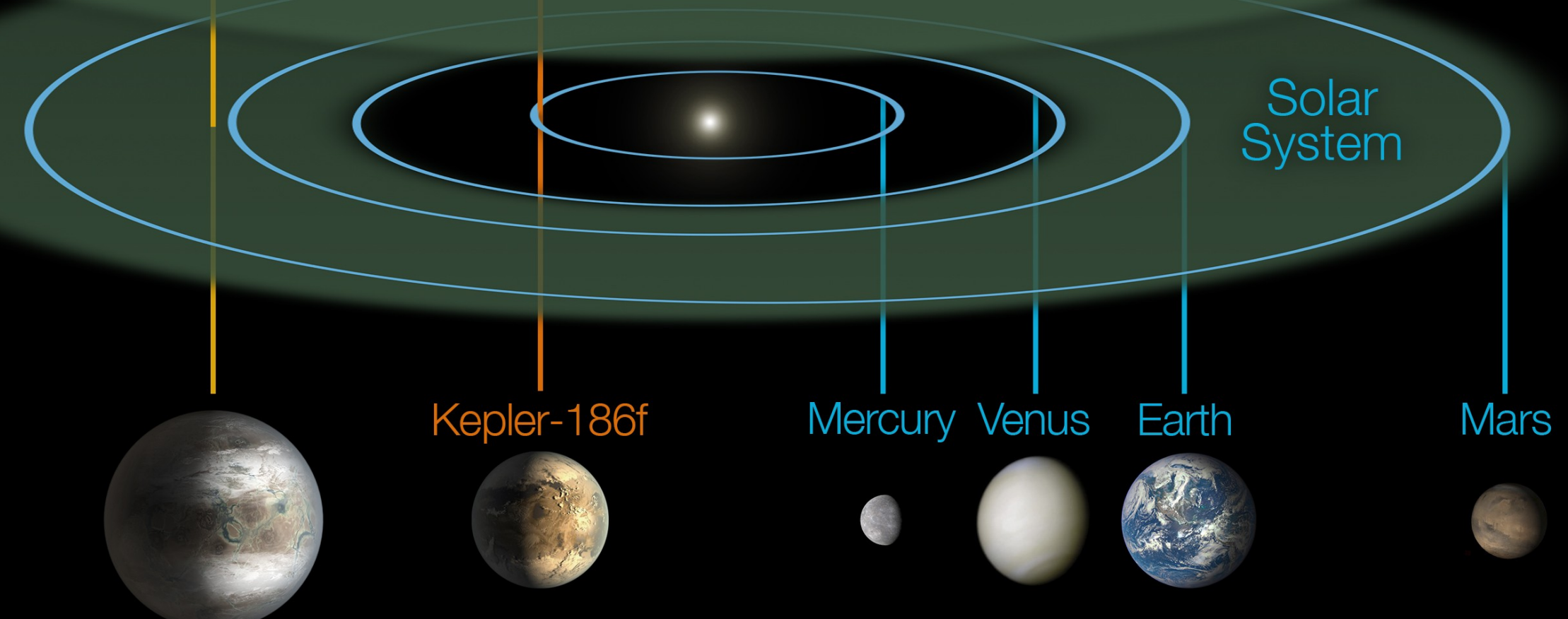

Kepler-452b

1400 ly away 


\section{Not Exoplanets -- Flare Stars}
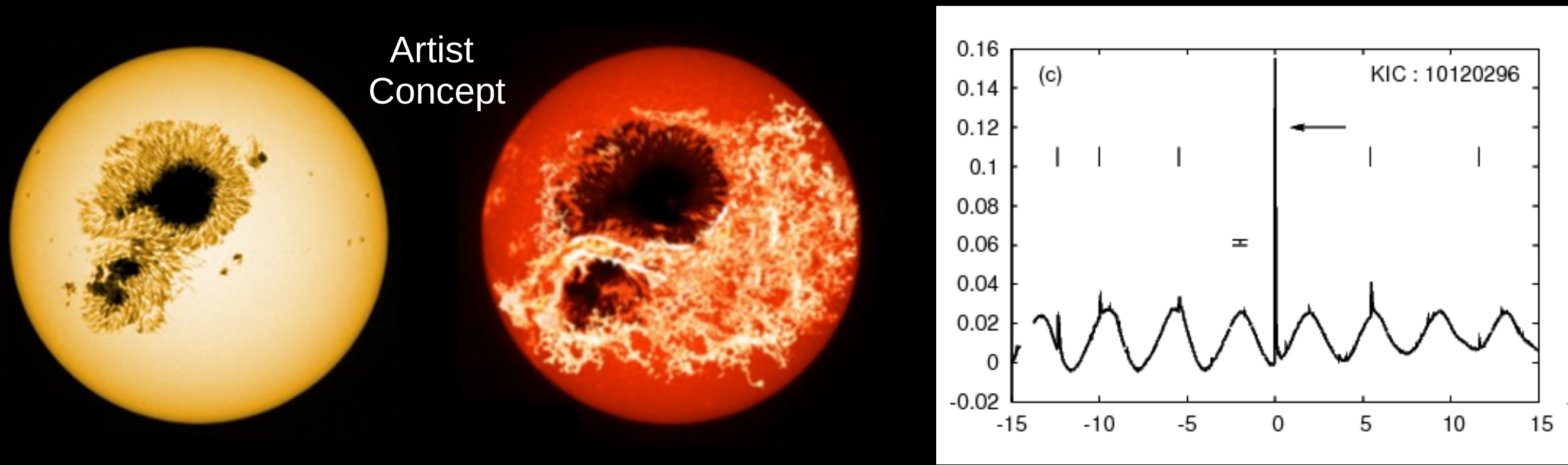

In a 2013 study, of stars with spectral classes ranging from F8 to G8, 1547 superflares were found on 279 solar-type stars. The most intense events increased the brightness of the stars by $30 \%$ and had an energy of $10^{36}$ ergs. White-light flares on the Sun change the brightness by about $0.01 \%$, and the strongest flares have a visible-light energy of about $10^{32}$ ergs.

Superflares on solar-type stars, Hiroyuki Maehara, et al., Nature, 2012

Flares common on all stars

Spots on flare stars are, in general, larger than on non-flare stars

Long flares from stars with low surface gravities

Flare energies correlated with stellar luminosity and radius

Flare stars across the $H-R$ diagram, L.A. Balona, in MNRAS, 2015 


\section{KIC 8462852: WTF object, Where's the Flux?}

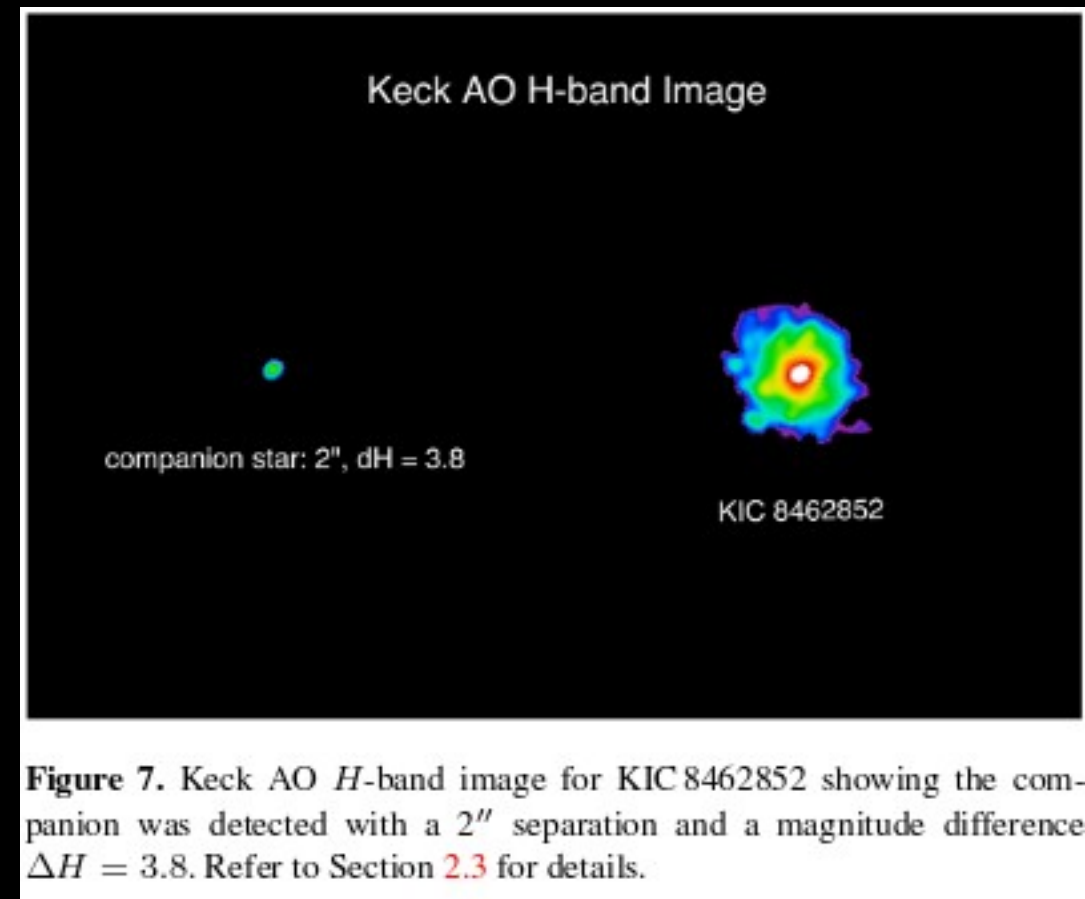

Younger star with coalescing material around it

Planetary debris field A cloud of disintegrating comets A megastructure
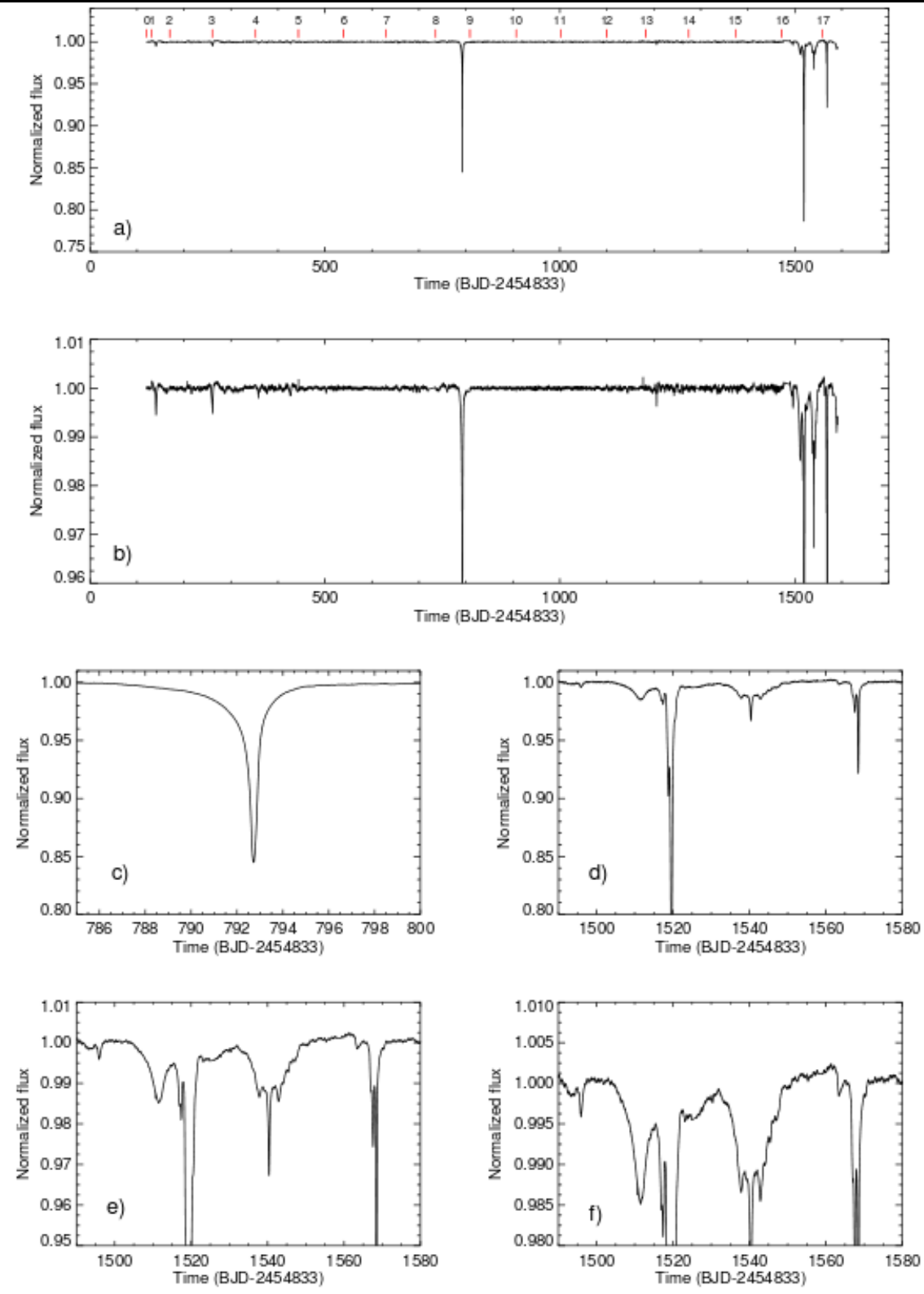

Figure 1. Montage of flux time series for KIC 8462852 showing different portions of the 4-year Kepler observations with different vertical scalings. The top two panels show the entire Kepler observation time interval. The starting time of each Kepler quarter is marked and labeled with a red vertical line in the top panel '(a)'. Panel '(c)' is a blowup of the dip near day 793, (D800). The remaining three panels, '(d)', '(c)', and '(f)', explore the dips which occur during the 90-day interval from day 1490 to day 1580 (D1 500). Refer to Section 2.1 for details. See Section 2.1 for detaik. 


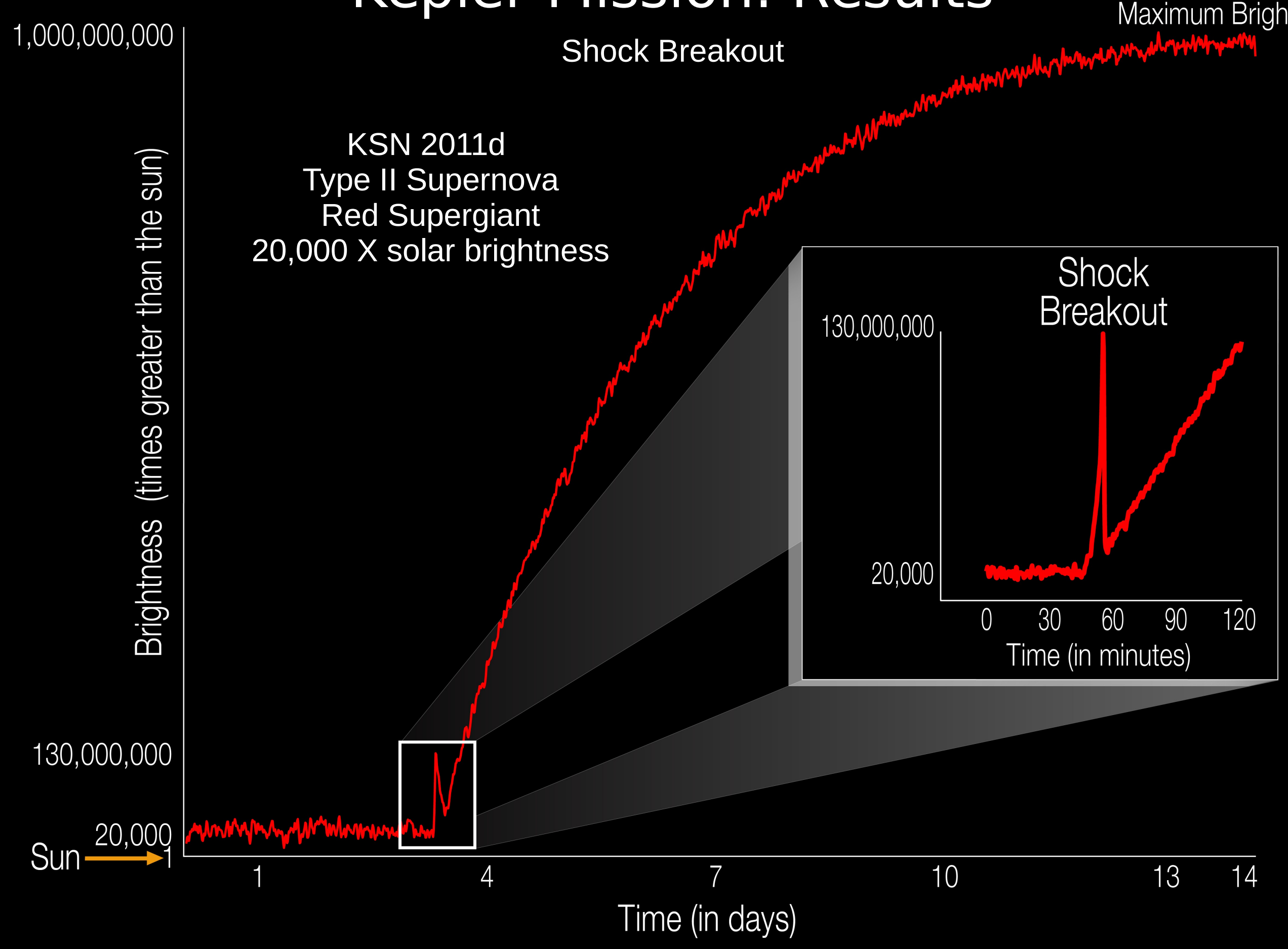




\section{New Validation Method}

Does the signal look like a planet?

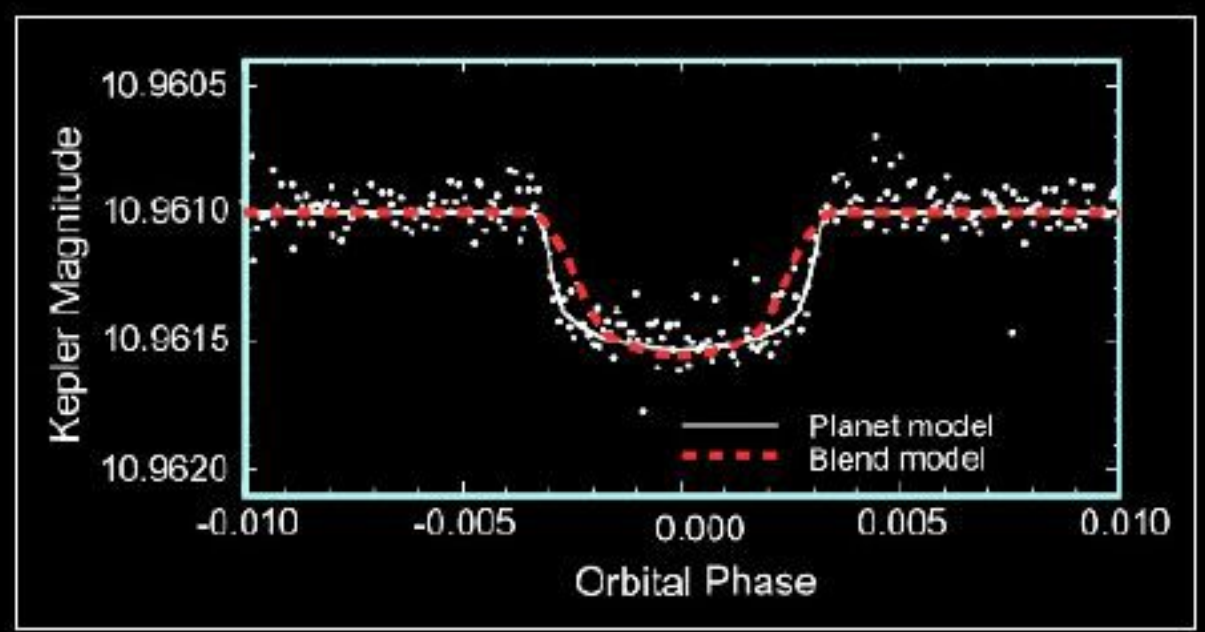

How common are imposters?

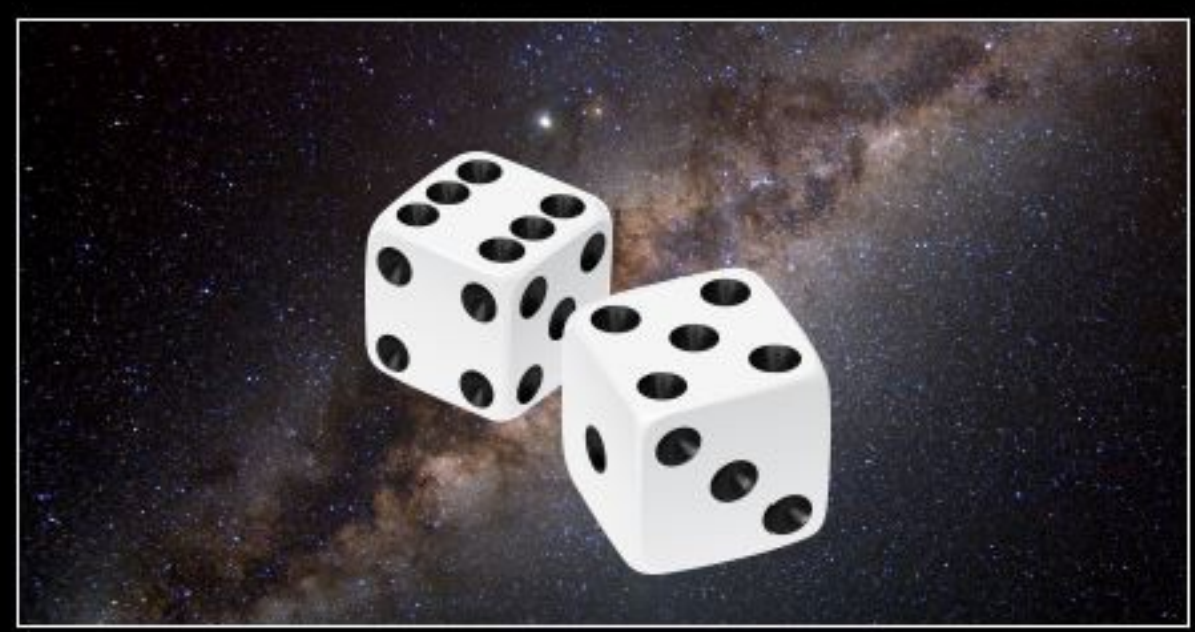

Results in ApJ paper: FALSE POSITIVE PROBABILITIES FOR ALL KEPLER OBJECTS OF INTEREST: 1284 NEWLY VALIDATED PLANETS AND 428 LIKELY FALSE POSITIVES; Timothy D. Morton, et al. http://www.astro.princeton.edu/ tdm/koi-fpp/ms.pdf 


\section{Exoplanet Discoveries Through the Years}

As of May 10, 2016

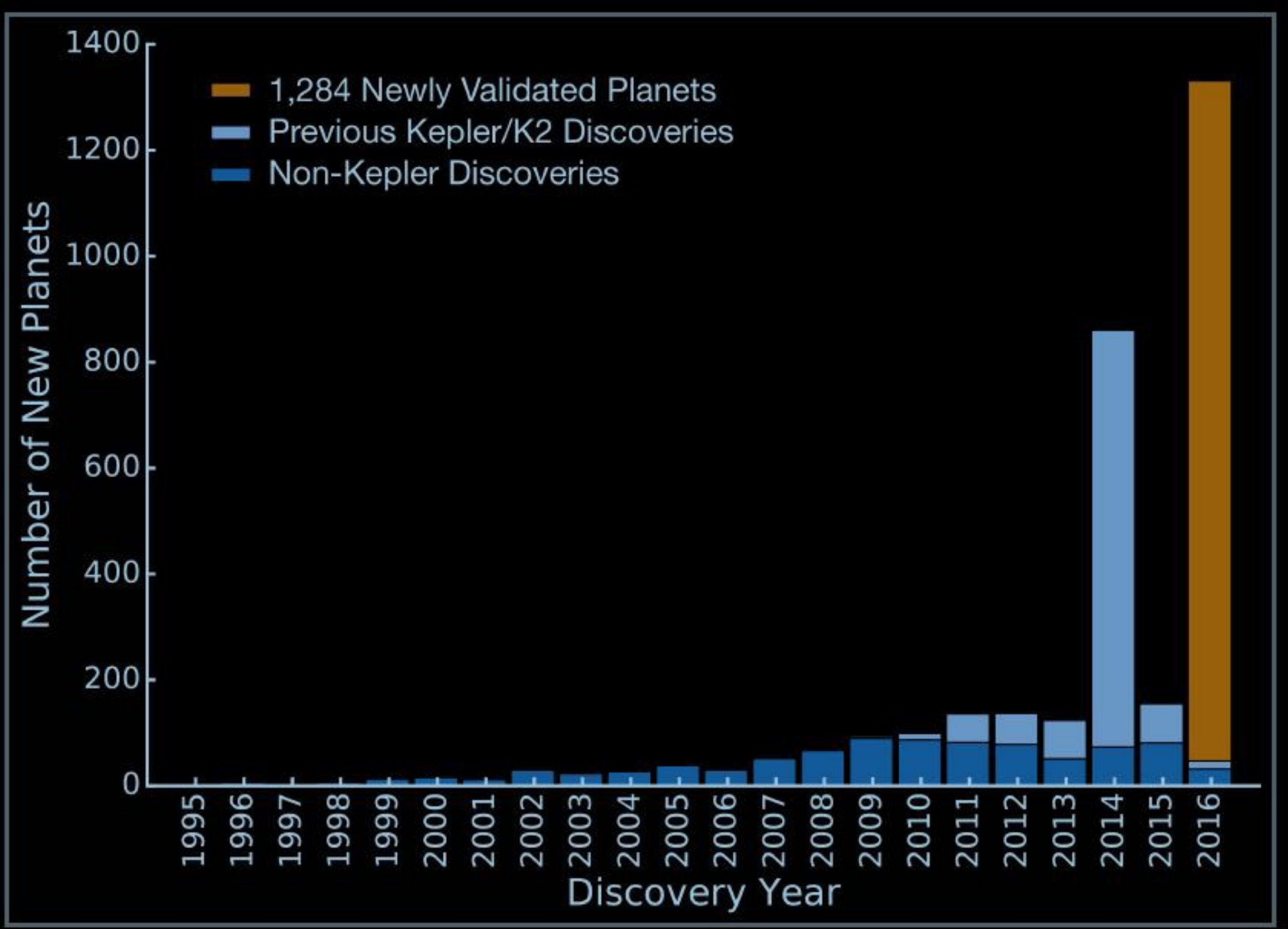




\section{Known Planets by Size}

As of May 10, 2016

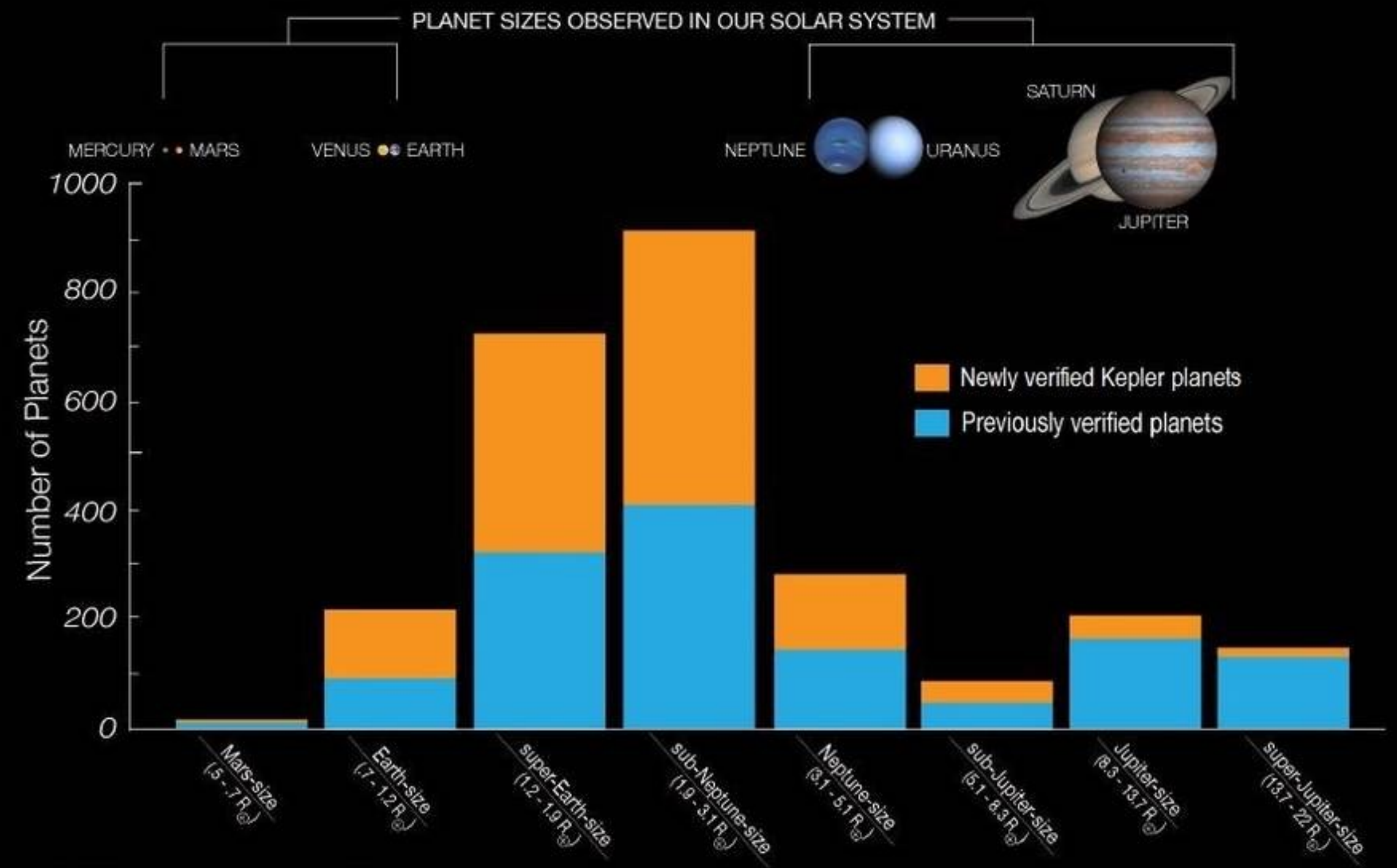




\section{Kepler's Small Habitable Zone Planets}

As of May 10, 2016

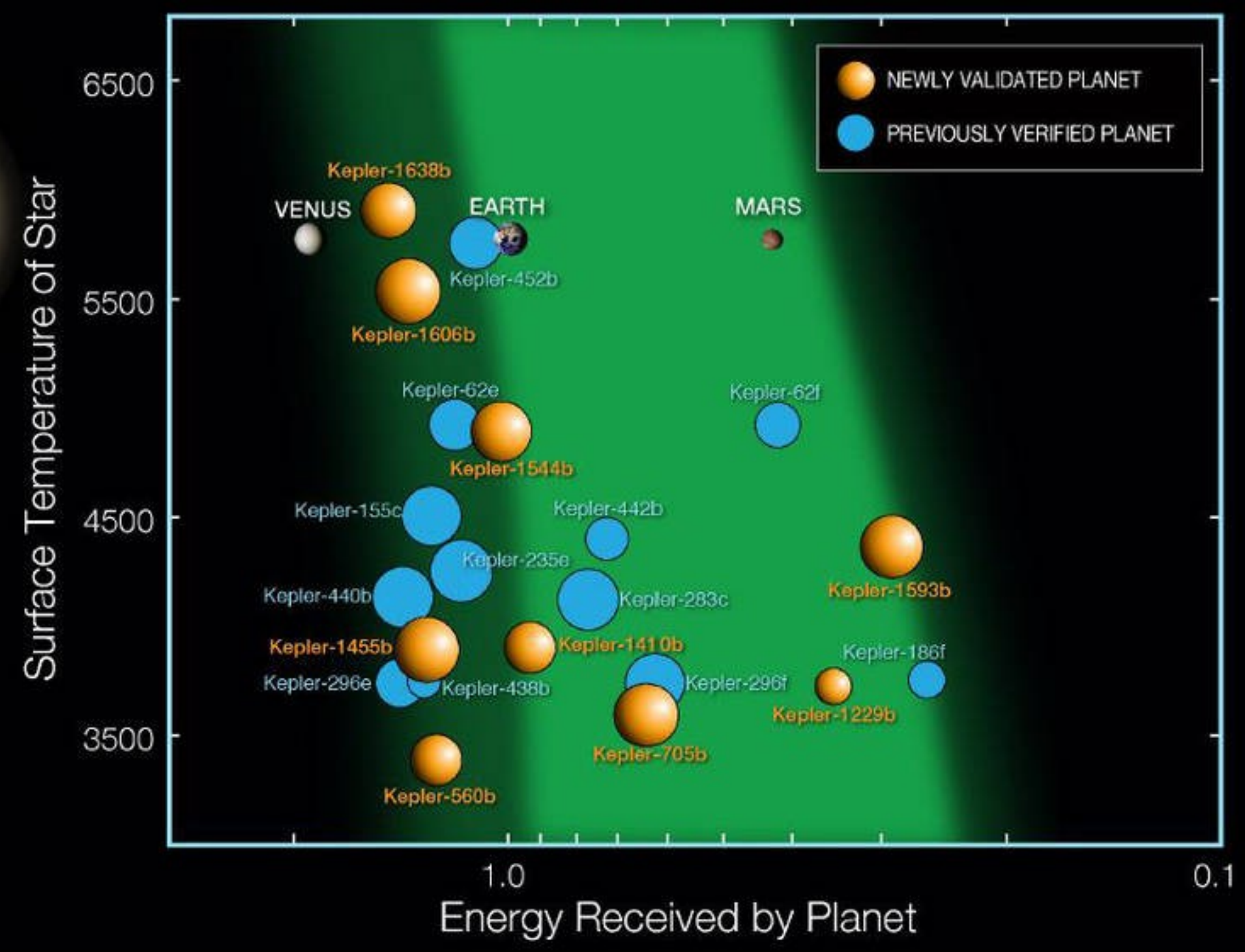

Closest Earth-like planet in habitable zone (statistically) at about 11 ly (Natalie Batalha during press conference May 10, 2016) 


\section{Kepler's Second Light: How K2 Will Work}

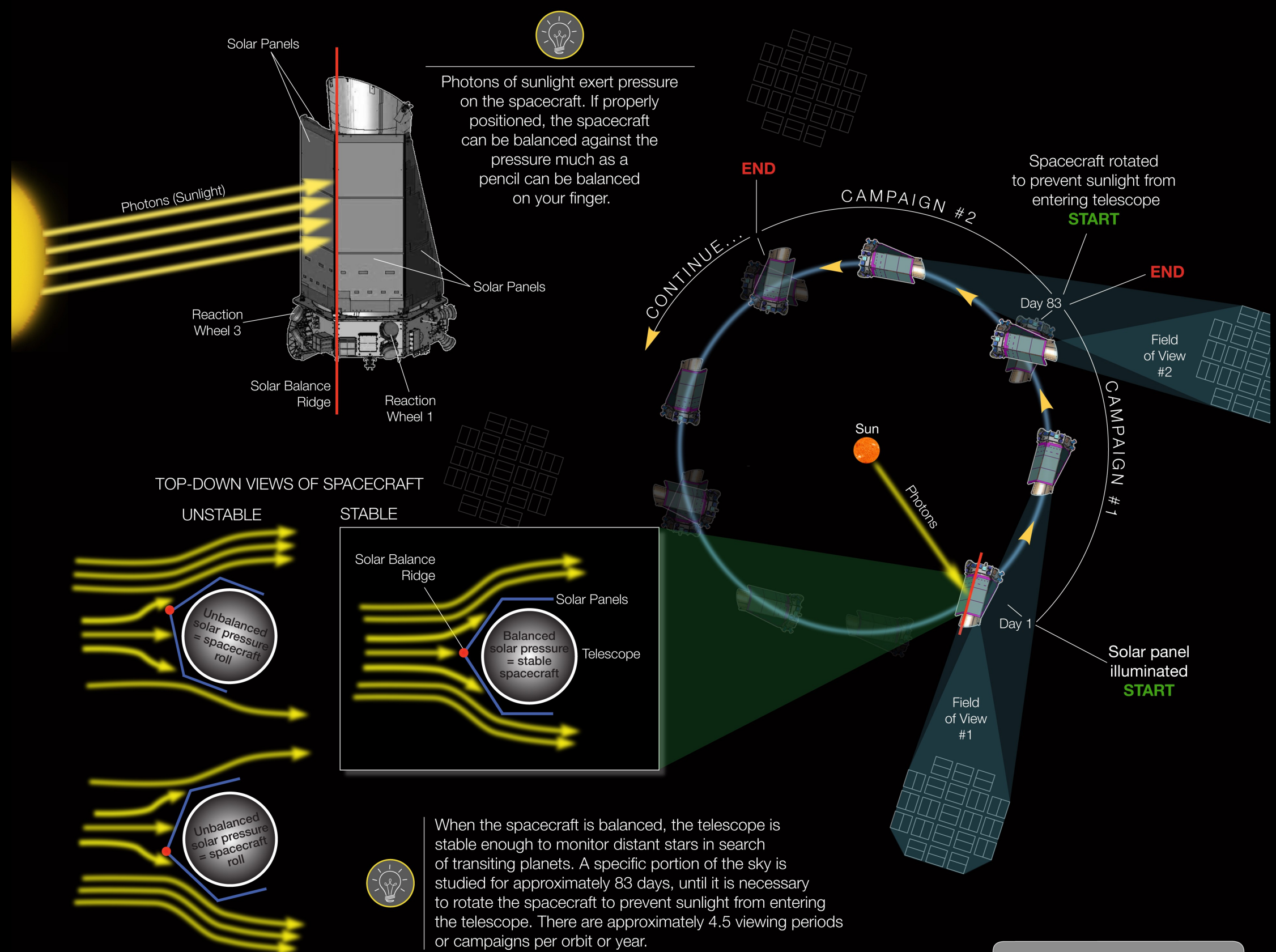




\section{Kepler Mission: Reconfiguring for Gravitational Lensing}

Along with ground-based observatories, $K 2$ will survey millions of stars in the direction of the center of the Milky Way for evidence of even more exoplanets.

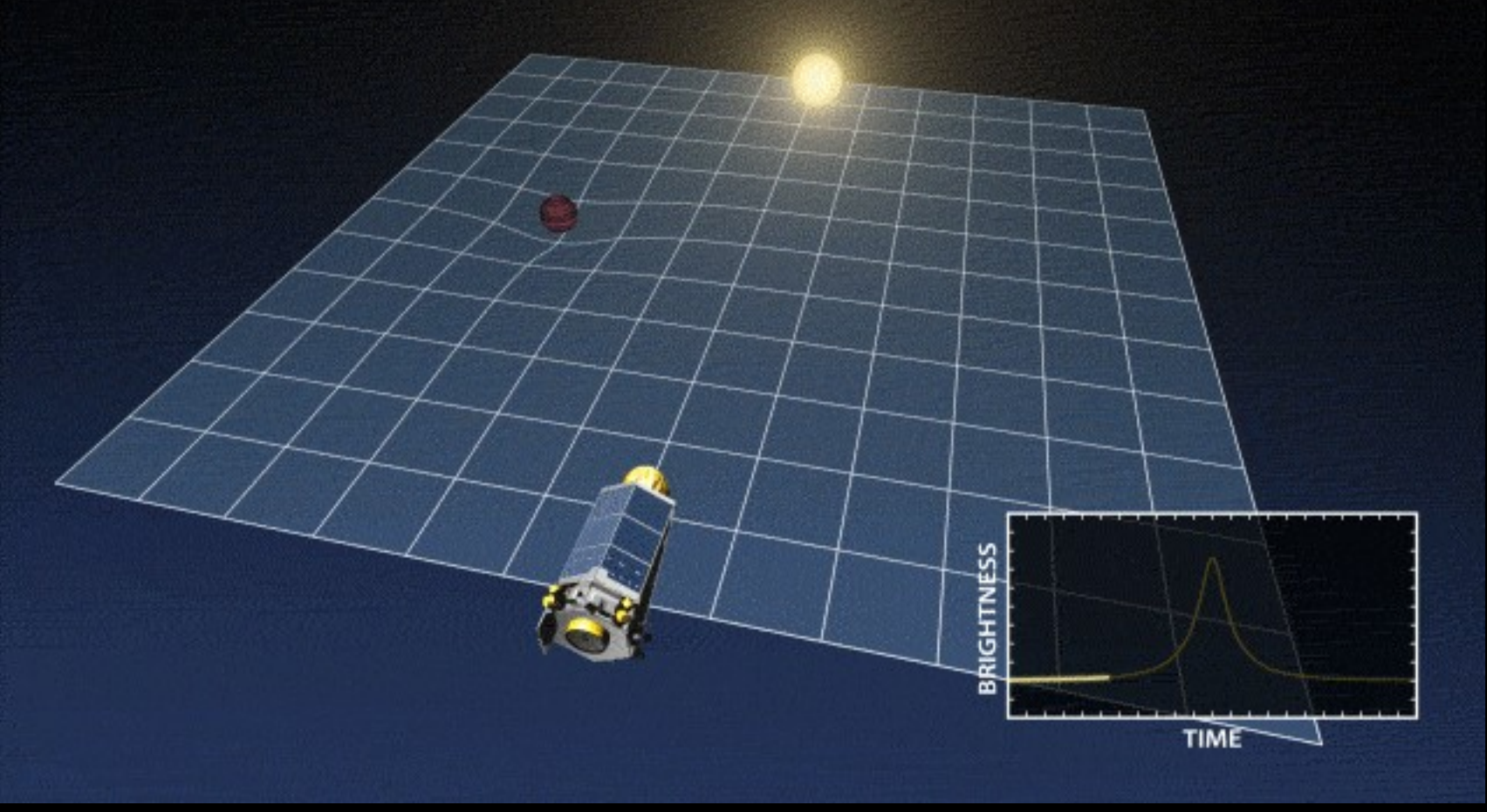


Exoplanet Missions

JWST

WFIRST

\section{TESS}

Kepler \&

K2

New Worlds

Telescope

Spitzer

Ground-based Observatories
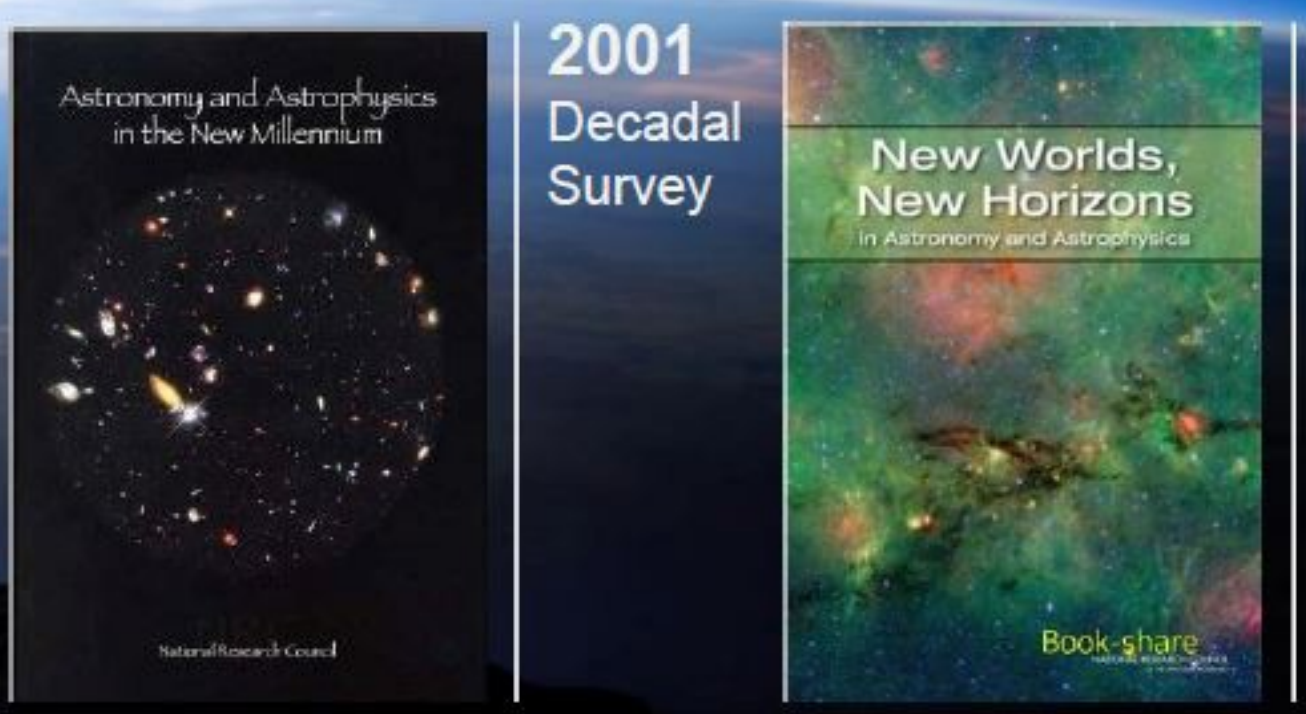

2010 Decadal Survey

Hubble
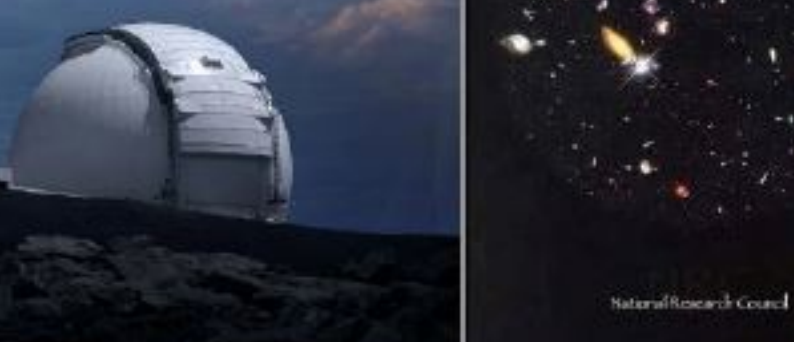


\section{Mission Perspective}

Kepler collected data for Approximately 2 more years of fuel

4 years and produced a remarkable data set that will be used for decades.

Together with other missions, Kepler and K2 are a part of this Arc of Discovery.

984 previously confirmed, 1284 newly validated

$100,000,000$ stars, $70 \%$ main sequence, $25 \%$ M stars...

The $\mathrm{K} 2$ mission is extending Kepler's legacy to new parts of the sky and new fields of study. 